\title{
Aggregate of Amphiphilic Block Copolymer as a Pseudo-Stationary Phase in Capillary Electrophoresis
}

\author{
Tohru Nakamura, Akira Ohki ${ }^{\dagger}$, Masaki Mishiro, Osamu Tsuyashima and Shigeru Maeda \\ Department of Applied Chemistry and Chemical Engineering, Faculty of Engineering, Kagoshima University, \\ Korimoto, Kagoshima 890-0065, Japan
}

\begin{abstract}
The use of an aggregate of amphiphilic block copolymer 1, which consists of poly[( $N$-acetylimino)ethylene $]$ and poly $[(N$ pentanoylimino)ethylene], for a pseudo-stationary phase in capillary electrophoresis has been examined. From gel-filtration chromatography, the aggregate from 1 (1-AG) was found to incorporate phenol. When the running solution contains 1-AG and sodium dodecyl sulfate (SDS), the electrophoretic mobility of phenols increases as the concentration of SDS is raised. In the absence of either 1-AG or SDS, the electrophoretic mobility becomes nearly zero. Thus, it is found that when 1-AG and SDS are added to the running solution, SDS is incorporated in 1-AG, giving negative charges on the 1AG, which works as a pseudo-stationary phase. By the addition of 1-AG and a low concentration of SDS in the running solution, six phenols are separated and the migration order of phenols is basically dependent on their lipophilicities. However, the migration order of some phenols is different from that in the micellar electrokinetic chromatography (MEKC) using a high concentration of SDS.
\end{abstract}

Keywords Amphiphilic block copolymer, aggregate, capillary electrophoresis, pseudo-stationary phase, phenols

Micelle-like polymer aggregates are a novel class of self-organized assemblies and provide functional fine particles, which sometimes act as hosts for various molecules. ${ }^{1-3}$ We have reported novel type amphiphilic block copolymers consisting of two poly $[(N$-acylimino)ethylene] blocks, which are different in hydrophilicity. ${ }^{4}$ The block copolymers can be easily prepared from 2-alkyl-2-oxazolines through two successive ring-opening polymerizations, since the polymerizations proceed in a living mechanism. We found that the block copolymers formed stable micelle-like aggregates with a diameter of $c a .100 \mathrm{~nm}$ in aqueous media. ${ }^{5-8}$ The block copolymer aggregates have a capability of incorporating various relatively hydrophobic compounds in aqueous media, similarly to normal micelles. The aggregates are also stable in aqueous-organic media, and thus the aggregates which encapsulate enzymes provide a utilization of enzyme in organic solutions. ${ }^{6-8}$ Also, the aggregate-enzyme hybrid has been used for preparing a biosensor membrane ${ }^{9}$ as well as the immobilization of enzymes on silica gel. ${ }^{10}$ Compared to the interaction of aggregates with proteins (enzymes), that

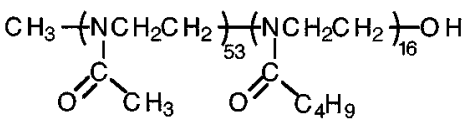

Block copolymer 1

\footnotetext{
† To whom correspondence should be addressed.
}

with small molecules has not yet been fully elucidated.

In the present study, we tried to use such an aggregate from a block copolymer 1, which consists of poly $[(N-$ acetylimino)ethylene] (hydrophilic part) and poly[( $N$ pentanoylimino)ethylene] (hydrophobic part), for a pseudo-stationary phase in capillary electrophoresis (CE). In CE, micellar electrokinetic chromatography (MEKC), in which a charged micelle is used for a pseudo-stationary phase, has been developed. These days MEKC is widely used in separations not only of ionic species, but also of neutral species. ${ }^{11,12}$ In MEKC, micelles from anionic surfactants, such as sodium dodecyl sulfate (SDS), have been mainly used. For pseudo-stationary phases other than micelles, some proteins ${ }^{13-15}$ and microemulsions ${ }^{16,17}$ have been used. However, to our knowledge, micelle-like polymer aggregates, such as the aggregate from 1 (1-AG), have not yet been applied to CE.

Block copolymer aggregates can provide a hydrophobic pool in aqueous media. ${ }^{2}$ It was reported that an anionic species, 8-anilino-1-naphthalene sulfonate, was effectively incorporated into block copolymer aggregates. ${ }^{5}$ Thus, it is anticipated that aggregates, such as 1-AG, are easily charged by the addition of a small amount of anionic surfactants. When such a negatively charged 1-AG is placed in a running solution in CE, 1AG will work as a pseudo-stationary phase, similarly to SDS micelles in MEKC. The migration of the pseudostationary phase (1-AG) may be controlled by adjusting the added amount of anionic surfactants, and the CE with 1-AG (AG-CE) will differ from normal MEKC in 
selectivity. Also, an examination of the interaction between 1-AG and various molecules by AG-CE may provide useful information for further applications of 1AG and related aggregates to various fields.

\section{Experimental}

Reagents

Block copolymer 1 was prepared by two-step block copolymerization with 2-methyl-2-oxazoline and 2butyl-2-oxazoline according to the literature. ${ }^{6}$ From a ${ }^{1} \mathrm{H}-\mathrm{NMR}$ analysis, the number-average molecular weight of 1 was estimated to be 6600 and the unit ratio of 1 was determined to be 53:16. Other chemicals were of reagent grade and used without further purification.

\section{Gel-filtration chromatography}

A Sephacryl S-500 HR column $(1.0 \times 35 \mathrm{~cm})$ was equilibrated and eluted with $50 \mathrm{mM}$ phosphate buffer $\left(\mathrm{Na}_{2} \mathrm{HPO}_{4}-\mathrm{KH}_{2} \mathrm{PO}_{4}, \mathrm{pH} 7.0\right)$ at room temperature. A sample was applied and eluted from the column in the equilibration buffer. Fractions $(0.8 \mathrm{ml})$ were collected at a flow rate of $8 \mathrm{ml} \mathrm{h}^{-1}$.

\section{Preparation of $\mathbf{1}-A G$}

The aggregate of $\mathbf{1}$ (1-AG) was obtained by stirring an aqueous solution containing $\mathbf{1}$ over night at room temperature, and free $\mathbf{1}$ was removed from the aggregate solution by ultrafiltration through a membrane (ADVANTEC UK-200). A dynamic light-scattering measurement was carried out using a COULTER N4SD submicrometer particle analyzer.

\section{Capillary electrophoresis}

All capillary electrophoresis experiments were carried out using an Otsuka Electronics CAPI-1000 under a voltage of $20 \mathrm{kV}$ at $25^{\circ} \mathrm{C}$. A fused-silica capillary column of $50 \mu \mathrm{m}$ i.d. with a column length of $70 \mathrm{~cm}$ (effective length, $50 \mathrm{~cm}$ ) was used, and detection was performed at $230 \mathrm{~nm}$ for 1-AG and $270 \mathrm{~nm}$ for phenols. The capillary was filled with a running solution of 20 $\mathrm{mM}$ phosphate buffer $\left(\mathrm{Na}_{2} \mathrm{HPO}_{4}-\mathrm{NaH}_{2} \mathrm{PO}_{4}, \mathrm{pH}\right.$ 7.0). Methanol was added to the sample solution as a marker of the electroosmotic flow.

\section{Results and Discussion}

\section{Incorporation of phenol by $\mathbf{1}-A G$}

To probe the interaction between 1-AG and a small neutral compound, such as phenol, gel-filtration chromatography was performed. Block copolymer $\mathbf{1}$ was dissolved in a phosphate buffer solution ( $\mathrm{pH}$ 7.0) and the solution was slowly stirred for $1 \mathrm{~d}$. Then, the resulting solution was placed in a Sephacryl S-500 HR column, which had been equilibrated with the buffer solution, at room temperature. The elution of $\mathbf{1}$ was monitored with a spectrophotometer at $230 \mathrm{~nm}$. The

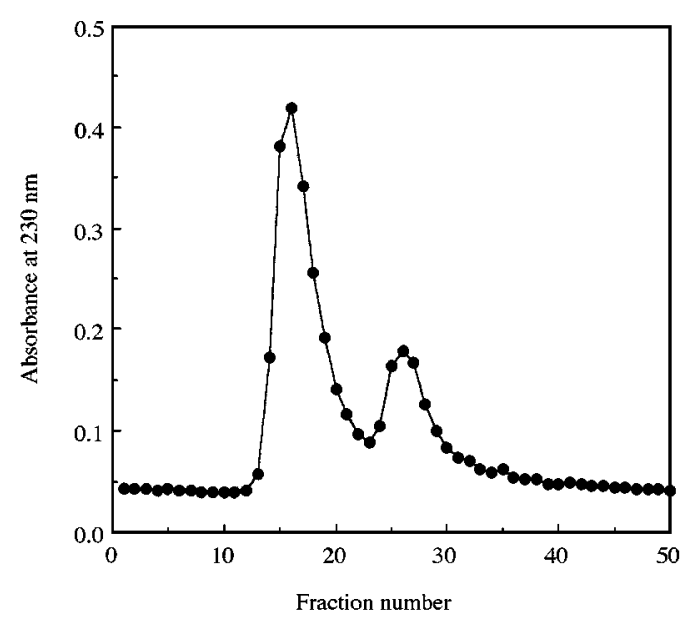

Fig. 1 Elution profile on gel-filtration chromatography for $\mathbf{1}$ in a $50 \mathrm{mM}$ phosphate buffer solution ( $\mathrm{pH} 7.0)$. The sample solution contained $3.0 \mathrm{mg} \mathrm{ml}^{-1}$ of $\mathbf{1}$.

elution profile is shown in Fig. 1, in which two peaks are observed at fraction numbers $13-22$ and $23-30$. It appears that the second peak can be ascribed to a molecularly dissolved chain of $\mathbf{1}$, because a homopolymer of poly[ $(N$-acetylimino $)$ ethylene $]\left(M_{\mathrm{n}}=5000\right)$ was eluted at the same location. The first peak should be due to the presence of higher molecular species, such as 1-AG. The particle size of 1-AG was determined to be $89 \mathrm{~nm}$ by dynamic light-scattering.

After the preparation of the 1-AG solution mentioned above, ultrafiltration through an ADVANTEC UK-200 membrane was carried out. Then, phenol was added to the solution, which was subsequently stirred for $2 \mathrm{~h}$. The resulting solution was placed in the same column at room temperature. Then, $\mathbf{1}$ and phenol were detected at $230 \mathrm{~nm}$ and $270 \mathrm{~nm}$, respectively; the elution profiles are indicated in Fig. 2. For the elution profile of $\mathbf{1}$ in Fig. 2, the second peak in Fig. 1 is almost extinguished, while the first peak is still observed. This result suggests that free $\mathbf{1}$ is almost removed by ultrafiltration and that $\mathbf{1 - A G}$ can stably exist in the absence of free $\mathbf{1}$. This behavior is quite different from that of normal micelles. The elution profile of phenol has a peak at the same place that the $1-A G$ peak exists, which means that phenol is almost incorporated in $\mathbf{1 - A G}$.

\section{Migration behavior of $1-A G$}

The migration behavior of 1-AG was examined in capillary-zone electrophoresis. As a sample solution, a phosphate buffer solution (20 mM, pH 7.0) containing $1.0 \mathrm{mg} \mathrm{ml} \mathrm{m}^{-1} \mathbf{1}-\mathrm{AG}$ (without free 1) and SDS was prepared. The sample solution was stirred for $2 \mathrm{~h}$ before injection. The detection of $\mathbf{1}$ in $\mathrm{CE}$ was performed at $230 \mathrm{~nm}$, at which SDS did not have absorbance. Figure 3 shows the effect of adding SDS in the sample solution upon the electrophoretic mobility $\left(\mu_{\mathrm{ep}}\right)$ of 1-AG. When SDS is not added, the electrophoretic mobility is almost zero because 1-AG itself is neutral under $\mathrm{pH}$ 7.0. The electrophoretic mobility increases with an 


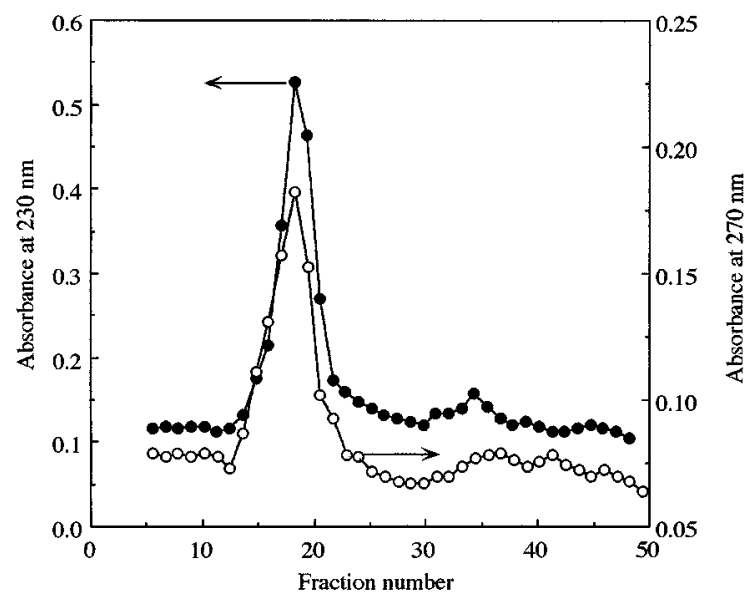

Fig. 2 Elution profile on gel-filtration chromatography for 1 and phenol in a $50 \mathrm{mM}$ phosphate buffer solution $(\mathrm{pH}$ 7.0). The sample solution contained $4.0 \mathrm{mg} \mathrm{ml}^{-1}$ of 1 and $10 \mathrm{mM}$ phenol.

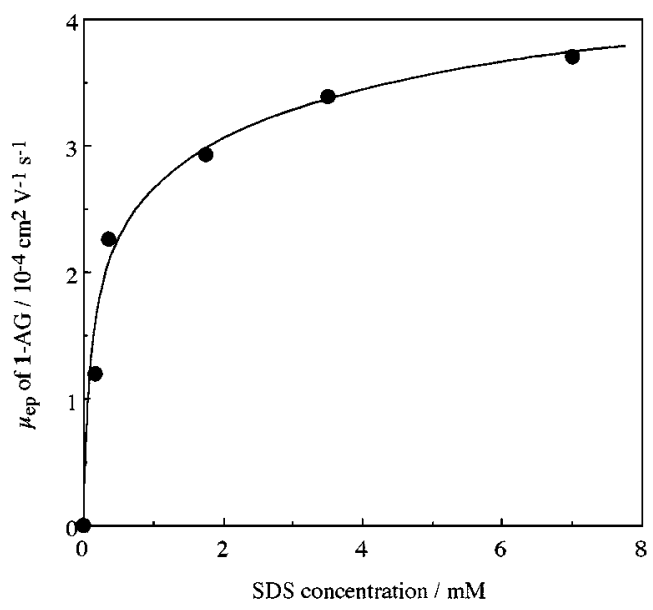

Fig. 3 Effect of adding SDS to the sample solution upon the electrophoretic mobility of 1-AG. The sample solution contained SDS, $1.0 \mathrm{mg} \mathrm{ml}^{-1}$ of $\mathbf{1}$, and $20 \mathrm{mM}$ phosphate buffer, while the running solution contained $20 \mathrm{mM}$ phosphate buffer (pH 7.0).

increase in the added amount of SDS. This result suggests that SDS is incorporated in 1-AG and provides negative charges on the surface of $\mathbf{1 - A G}$ in the sample solution. The incorporation continues during the migration, and thus 1-AG behaves like an anionic micelle in aqueous media when a low concentration of SDS, for which SDS itself does not make micelle so much, is added.

Negatively charged 1-AG as a pseudo-stationary phase

One can assume that when negatively charged 1-AG is present in the running solution, the aggregate will work as a pseudo-stationary phase, as shown in Fig. 4 (AG-CE). To probe the possibility of AG-CE, the migration behavior of phenol was examined. As can be seen in Table 1, when both 1-AG and SDS are present

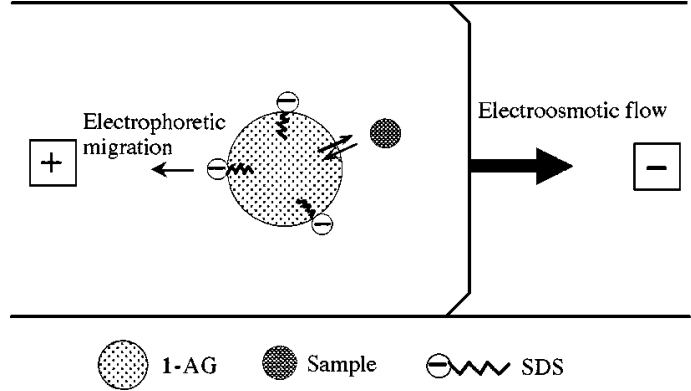

Fig. 4 Schematic representation of AG-CE.

Table 1 Electrophoretic mobility of phenols

\begin{tabular}{|c|c|c|c|}
\hline \multirow{2}{*}{ Sample } & \multicolumn{2}{|c|}{ Running solution ${ }^{\mathrm{a}}$} & \multirow{2}{*}{$\mu_{\mathrm{ep}} / 10^{-4} \mathrm{~cm}^{2} \mathrm{~V}^{-1} \mathrm{~s}^{-1}$} \\
\hline & 1-AG/mg ml ${ }^{-1}$ & $\mathrm{SDS} / \mathrm{mM}$ & \\
\hline \multirow[t]{4}{*}{ Phenol } & 0 & 0 & $\mathrm{~b}$ \\
\hline & 0 & 7.0 & $\mathrm{~b}$ \\
\hline & 1.0 & 0 & $\mathrm{~b}$ \\
\hline & 1.0 & 7.0 & 0.56 \\
\hline \multirow[t]{4}{*}{$o$-Cresol } & 0 & 0 & $\mathrm{~b}$ \\
\hline & 0 & 7.0 & $\mathrm{~b}$ \\
\hline & 1.0 & 0 & $\mathrm{~b}$ \\
\hline & 1.0 & 7.0 & 1.20 \\
\hline \multirow[t]{4}{*}{ 1-Naphthol } & 0 & 0 & $\mathrm{~b}$ \\
\hline & 0 & 7.0 & $\mathrm{~b}$ \\
\hline & 1.0 & 0 & $\mathrm{~b}$ \\
\hline & 1.0 & 7.0 & 2.75 \\
\hline
\end{tabular}

a. The solution contained $20 \mathrm{mM}$ phosphate buffer (pH7.0). b. Nearly zero.

in the running solution, the $\mu_{\text {ep }}$ value of phenol is 0.56 , whereas the value becomes almost zero in the absence of either 1-AG or SDS. When the migration behaviors of $o$-cresol and 1-naphthol were tested under the same conditions, similar results were obtained (Table 1). Thus, only when both 1-AG and SDS are present in the running solution, the electrophoretic mobility is observed. Therefore, it is concluded that $\mathbf{1 - A G}$, which is given negative charges by the addition of SDS, favorably works as a pseudo-stationary phase in CE.

Figure 5 shows the effect of the SDS concentration in the running solution upon the electrophoretic mobility of phenols. For the three phenols (phenol, $o$-cresol, and 1-naphthol) the electrophoretic mobility increases with an increase in the SDS concentration. This result supports the idea depicted in Fig. 4. As the SDS concentration is raised, more SDS molecules are incorporated in 1-AG, which results in an increase in the negative charge on 1-AG, and thus the migration of 1-AG incorporating phenols is retarded. The electrophoretic mobility of phenols decreases in the order 1-naphthol $>o$-cresol>phenol, which may parallel their lipophilicities. This result suggests that more lipophilic compounds tend to more distribute into $\mathbf{1 - A G}$, which leads to an increase in the electrophoretic mobility. 


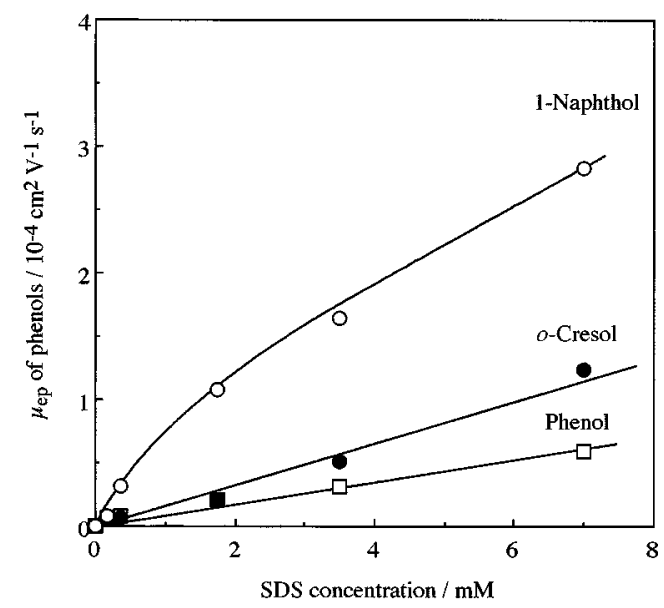

Fig. 5 Effect of adding SDS to the running solution upon the electrophoretic mobility of phenols. The running solution contained SDS, $1.0 \mathrm{mg} \mathrm{ml}^{-1}$ of $1-\mathrm{AG}$, and $20 \mathrm{mM}$ phosphate buffer ( $\mathrm{pH} 7.0)$.

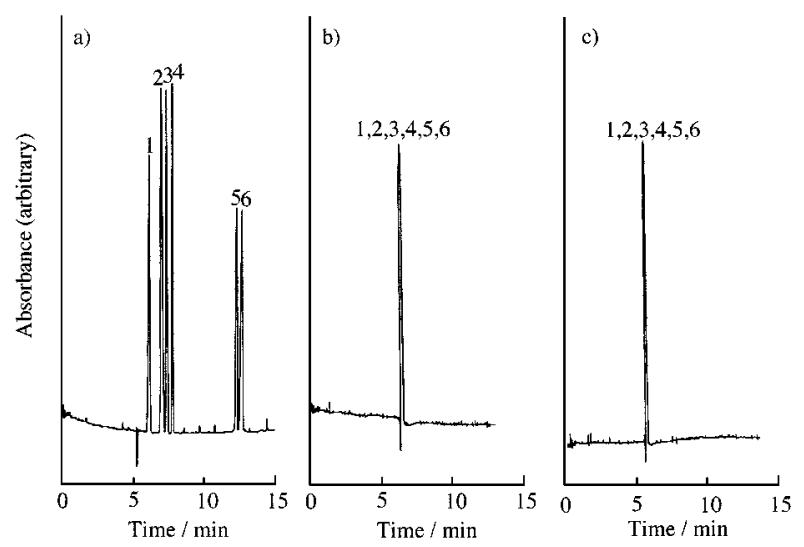

Fig. 6 Electropherogram of phenols. Samples: 1, phenol; 2, $m$-cresol; 3, $o$-cresol; 4, $p$-cresol; 5, 2-naphthol; 6, 1-naphthol. Running solution: a) $1.0 \mathrm{mg} \mathrm{ml}^{-1} \mathbf{1}-\mathrm{AG}, 7.0 \mathrm{mM}$ SDS, $20 \mathrm{mM}$ phosphate buffer (pH 7.0). b) Same as a) without 1-AG. c) Same as a) without SDS.

\section{Separation of phenols}

Figure 6a shows an electropherogram of six phenols when a running solution containing $1.0 \mathrm{mg} \mathrm{ml}^{-1} \mathbf{1}-\mathrm{AG}$, $7.0 \mathrm{mM}$ SDS, and $20 \mathrm{mM}$ phosphate buffer $(\mathrm{pH} 7.0)$ is used. The six phenols are separated and the migration time decreases in the order 1-naphthol>2-naphthol>pcresol $>O$-cresol $>m$-cresol $>$ phenol. When either $\mathbf{1}$-AG or SDS is absent in the running solution, the electropherogram gives only one peak, and the electrophoretic mobilities of these phenols are almost zero (Figs. 6b and $6 c)$.

The effect of the $\mathrm{pH}$ in the running solution upon the migration behavior of phenols is indicated in Fig. 7. When a pH of 5.0 (20 mM phthalate buffer) is used, the migration of phenols is slower than when the $\mathrm{pH}$ is 7.0 (Fig. 6a); however the migration order and the separation of phenols are not changed. On the contrary, the

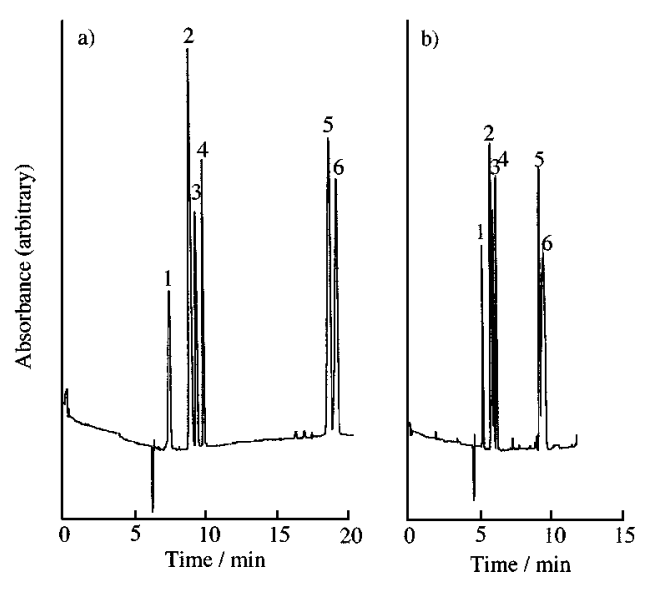

Fig. 7 Electropherogram of phenols. The samples are the same as those in Fig. 6. Running solution: a) $1.0 \mathrm{mg} \mathrm{ml}^{-1} \mathbf{1}$ -

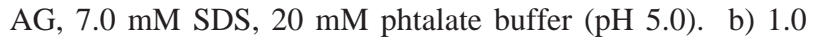
$\mathrm{mg} \mathrm{ml}^{-1}$ 1-AG, 7.0 mM SDS, $20 \mathrm{mM}$ borate buffer ( $\mathrm{pH} 9.0$ ).

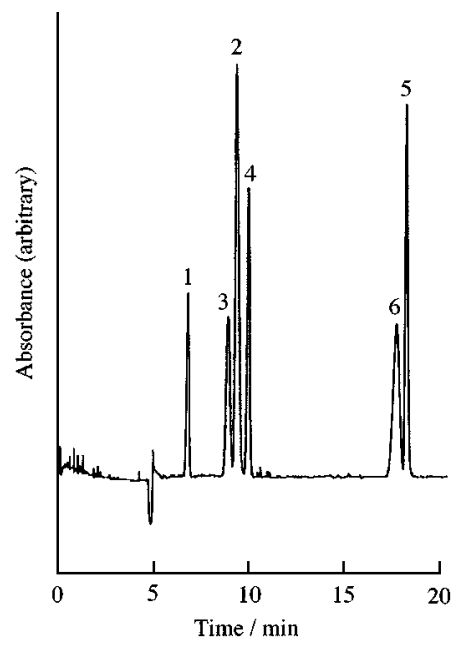

Fig. 8 Electropherogram of phenols. The samples are the same as those in Fig. 6. Running solution: 50 mM SDS, 20 $\mathrm{mM}$ phosphate buffer ( $\mathrm{pH} 7.0$ ).

use of a $\mathrm{pH}$ of 9.0 (20 $\mathrm{mM}$ borate buffer) results in a decrease in the migration time, and the separation of phenols is considerably worse than when the $\mathrm{pH}$ is 7.0.

In Table 2, the electrophoretic mobilities of six phenols under different $\mathrm{pH}$ conditions are listed. The electrophoretic mobilities of six phenols when the $\mathrm{pH}$ is 5.0 are slightly smaller than those when the $\mathrm{pH}$ is 7.0. This result suggests that the considerable retardation in the migration time observed when the $\mathrm{pH}$ is 5.0 (see in Fig. 7) is mainly due to a decrease in the electroosmotic flow caused by the lowering of $\mathrm{pH}$ in the running solution. However, when the $\mathrm{pH}$ is 9.0, the electrophoretic mobilities of six phenols increase, suggesting that the phenols are partially deprotonated to produce anionic species. The anionic species, themselves, undergo electrophoretic migration, and thus the electrophoretic mobilities of phenols increase, although the distribution 
Table 2 Electrophoretic mobility of phenols

\begin{tabular}{ccccccc}
\hline & \multicolumn{5}{c}{$\mu_{\mathrm{ep}} / 10^{-4} \mathrm{~cm}^{2} \mathrm{~V}^{-1} \mathrm{~s}^{-1}$} \\
\cline { 2 - 5 } & Phenol & $m$-Cresol & $o$-Cresol & $p$-Cresol & 2-Naphthol & 1-Naphthol \\
\hline 5.0 & 0.66 & 1.28 & 1.52 & 1.67 & 3.09 & 3.13 \\
7.0 & 0.69 & 1.28 & 1.56 & 1.69 & 3.13 & 3.20 \\
9.0 & 0.79 & 1.50 & 1.61 & 1.83 & 3.41 & 3.47 \\
\hline
\end{tabular}

a. The running solution conditions for $\mathrm{pH}=5.0,7.0$, and 9.0 are described in Fig. 7a, Fig. 6a, and Fig. 7b, respectively.

of phenol into 1-AG may be reduced.

\section{Comparison with SDS-MEKC}

In conventional MEKC, SDS micelle is mainly used. Figure 8 shows an electropherogram of the six phenols when a running solution containing $50 \mathrm{mM}$ SDS and 20 $\mathrm{mM}$ phosphate buffer ( $\mathrm{pH}$ 7.0) is used. In this condition, SDS, itself, makes an anionic micelle which works as a pseudo-stationary phase (SDS-MEKC). The six phenols are separated and the migration time decreases in the order 2-naphthol $>1$-naphthol $>p$ cresol $>m$-cresol $>O$-cresol $>$ phenol. Compared with the result when AG-CE is performed (Fig. 6a), the order of $m$-cresol and $o$-cresol and that of 2-naphthol and 1naphthol are reversed.

It appears that 1-AG has susceptibility for a hydrogen-bonding interaction within the aggregate because of the presence of amide-oxygen. However, the SDS micelle does not have such an interaction in its core. Apparently, $o$-cresol is worse than $m$-cresol in terms of the hydrogen-bond donor due to a steric hindrance by the presence of the $o$-methyl group, while the hydrogen-bonding ability of 1-naphthol may be superior to that of 2-naphthol by considering those acidities. Thus, the above-mentioned selectivity change may be rationally explained by a consideration of hydrogen-bonding interaction, by which 1-AG has a high affinity to $m$ cresol and 1-naphthol compared to the SDS micelle.

In conclusion, it has been proved that a block copolymer aggregate, 1-AG, favorably works as a pseudo-stationary phase in $\mathrm{CE}$ when the aggregate is negatively charged by the addition of a small amount of anionic surfactants. The migration order of phenols is basically dependent on their lipophilicities, which is similar to that when SDS-MEKC is performed. However, the migration order of some phenols in AG-CE is different from that in SDS-MEKC. For the block copolymers, such as $\mathbf{1}$, it is easy to change the hydrophilicity/hydrophobicity balance and to introduce some moieties to the side chain, which will produce new selectivities. Also, the block copolymer aggregates can incorporate not only small molecules, but also bio-polymers, such as proteins.
Thus, the utilization of the block copolymer aggregates, such as 1, in CE should be worth further exploration.

The authors are grateful to Dr. K. Naka of Kyoto University for valuable suggestions about polymer aggregate. This work was supported by a Grant-in-Aid for Scientific Research (C) No. 10650799 from the Ministry of Education, Science, Sports, and Culture, Japan.

\section{References}

1. T. N. Khan, R. H. Mobbs, C. Price and J. R. Quintana, Eur. Polym. J., 23, 191 (1987)

2. M. Wilhelm, C. L. Zhao, Y. Wang, R. Xu and M. Winnik, Macromolecules, 24, 1033 (1991).

3. M. Li, M. Jiang, Y. Zhang and Q. Fang, Macromolecules, 30, 470 (1997)

4. K. Naka, A. Ohki and S. Maeda, Chem. Lett., 1991, 1303.

5. K. Naka, Y. Kubo, A. Ohki and S. Maeda, Polymer J., 26, 243 (1994)

6. K. Naka, T. Nakamura, A. Ohki and S. Maeda, Polymer J., 27, 1071 (1995)

7. K. Naka, T. Nakamura, A. Ohki and S. Maeda, Macromol. Chem. Phys., 198, 101 (1997).

8. K. Naka, T. Nakamura, A. Ohki and S. Maeda, J. Macromol. Sci. A, 34, 587 (1997).

9. A. Ohki, K. Naka, O. Ito and S. Maeda, Chem. Lett., 1994, 1065.

10. K. Naka, N. Kitano, K. Iwaki, A. Ohki and S. Maeda, Chem. Lett., 1995, 1037.

11. M. G. Khaledi, J. Chromatogr. A, 780, 3 (1997)

12. P. G. Muijselaar, K. Otsuka and S. Terabe, J. Chromatogr. A, 780, 41 (1997)

13. G. E. Baker, P. Russo and R. A. Hartwick, Anal. Chem., 64, 119 (1993).

14. S. Busch, J. C. Kraak and H. Poppe, J. Chromatogr., 635, 119 (1993)

15. Y. Ishihama, Y. Oda, N. Asakawa, Y. Yoshida and T. Sato, J. Chromatogr. A, 666, 193 (1994).

16. H. Watarai, Chem. Lett., 1991, 391.

17. S. Terabe, N. Matsubara, Y. Ishihama and Y. Okada, J. Chromatogr., 608, 23 (1992).

(Received February 25, 1999) (Accepted May 26, 1999) 